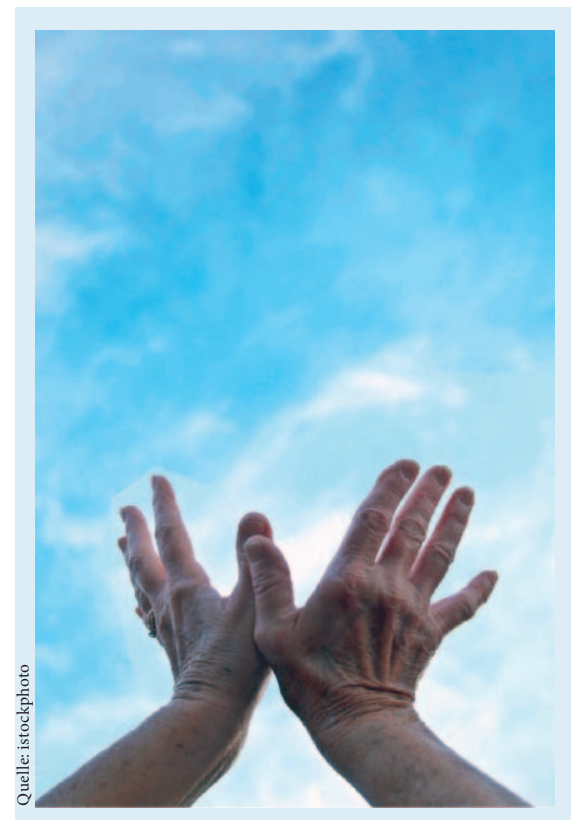

\section{Rheumatologie}

Rheuma ist längst zu einer Volkskrankheit geworden, an der die zunehmend älter werdende Bevölkerung leidet. Weil die Schulmedizin rasch an ihre Grenzen gerät, suchen viele Hilfe bei den Methoden der Ganzheitsmedizin. Was sie leisten kann, erfahren Sie in den Expertengesprächen ab Seite 317. Welchen Einfluss eine gesündere Ernährung auf die Beschwerden hat und wie sie zur Prophylaxe beitragen kann, steht auf Seite 324. Über den Einsatz von Phytotherapeutika können Sie sich direkt nebenan informieren. Dort lesen Sie auch, dass die Cochrane-Kollegen von der wissenschaftlichen Datenlage zur Wirkung der Pflanzenwirkstoffe eher enttäuscht sind.

\title{
Bei Rheuma auf die Phytotherapie setzen
}

Bei rheumatischen Erkrankungen greifen viele Patienten zu phytotherapeutischen Mitteln. Theoretisch ist ihre Wirkung geklärt, aber nur in wenigen Fällen gesichert.

Die Prozesse, die zu Entzündungen führen, sind auf molekularer Ebene gut geklärt und schon bis ins Detail verstanden. Damit hat sich auch die Kenntnis der Vorgänge verbessert, die $\mathrm{zu}$ entzündlichen Formen des Rheumas führen. Diese Fortschritte haben in der Vergangenheit zur Entwicklung einer Reihe von synthetischen Schmerzmitteln und entzündungshemmenden Substanzen beigetragen, die direkt in die biochemischen und molekularen Abläufe eingreifen, die Entzündungen entstehen lassen.

Viele der synthetischen Schmerzmittel greifen in die Cyclooxygenase(Cox)-2-Inhibitoren-Steuerung ein, die bei Entzündungen eine wichtige Rolle spielt. Das scheint ein vielversprechender Mechanismus zu sein, der auch für den phytotherapeutischen Ansatz Potenzial besitzt.

Bei den Pflanzen, die im Zuge der Phytotherapie eingesetzt werden, lässt sich die Wirkung auf Entzündungsprozesse grob in drei Wirkungsmechanismen, die einen Einfluss haben, unterscheiden [1]. In der ersten Gruppe sind Pflanzen, deren Substanzen auf die Biosynthese von Eicosanoiden wirken. Darauf ist der Effekt der Extrakte aus Teufelskralle (Harpagophytum procumbens) zurückzuführen. Die zweite Gruppe umfasst Pflanzen, deren Inhaltsstoffe auf die Gamma-Linolensäure-Stoffwechselprozesse wirken. Sie sorgen so für eine Reduktion der Synthese von Interleukin-Alpha oder induzieren die pro-Interleukin-1-Beta-Gen-Expression. Sie hemmen die Produktion von
Prostaglandinen und Leukotrienen. In der dritten Gruppe finden sich Pflanzen, wie etwa das Mutterkraut (Tanacetum parthenium), die Plättchenaggregation hemmen. Die Extrakte vieler Heilpflanzen wirken allerdings auf mehr als eine Weise. So greifen die Wirkstoffe des Mutterkrauts auch in die Prostaglandinsynthese und die Bildung von Leukotrienen ein.

Während der letzten 10-15 Jahre sind vermehrt pflanzliche Wirkstoffe untersucht worden, von denen man annahm, dass sie ebenfalls auf molekularer Ebene in die Entstehung von Entzündungen eingreifen könnten. In klinischen Studien wurde ihre Einsatzmöglichkeit bei der Behandlung von rheumatischen Erkrankungen geprüft. Obwohl es eine ganze Reihe von Pflanzenarten gibt, deren Inhaltsstoffe eine antiinflammatorische Wirkung haben könnten, ist die wissenschaftliche Datenbasis für die klinische Wirkung der verschiedenen Phytotherapeutika bei rheumatischen Erkrankungen äusserst dünn. Nur für die wenigsten Wirkstoffe findet sich eine genügend grosse Zahl von Studien, die es zulässt, über die Wirksamkeit eine gesicherte Aussage zu machen. "Eine Reihe von Therapien mit Pflanzenstoffen ist nur unzureichend gerechtfertigt, da sie nur durch eine Studie oder nicht vergleichende Studien belegt sind», schreiben die Autoren einer Cochrane-Studie [2] über die Wirksamkeit von Pflanzenwirkstoffen bei der rheumatischen Arthritis.

\section{KARGER}


In dieser Cochrane-Studie konnten aus der gesamten wissenschaftlichen Literatur nur insgesamt 22 Studien analysiert werden, um die Wirksamkeit der Phytotherapie zu untersuchen. Kriterium war dabei der Vergleich zu Placebo oder Kontrollen bei Patienten, die unter rheumatischer Arthritis leiden. Bei 7 Studien konnte eine leichte Besserung von Schmerzen und eine Reduktion der Bewegungsbehinderung festgestellt werden.

Entscheidend dafür war das Vorhandensein von Gamma-Linolensäure, etwa aus Extrakten der Nachtkerze (Oenothera), aus den Samen von Borretsch-Gewächsen oder aus den Früchten von Johannisbeeren [2]. Bei Dosierungen von $\geq 1400 \mathrm{mg}$ pro Tag liess sich eine Wirkung zeigen und eine Linderung der rheumatischen Beschwerden feststellen. Eine Dosierung von $<500 \mathrm{mg}$ pro Tag scheint nicht wirksam zu sein [2].

Die Teufelskralle enthält eine ganze Reihe von Inhaltsstoffen, die in Entzündungsprozesse des Körpers eingreifen. Die Pflanze, die zu den Sesamgewächsen gehört, wächst auf dem Boden liegend und weist bis 1,5 Meter lange Ausläufer auf, in deren Blattachseln die roten Trompetenblüten stehen. Die Teufelskralle wirkt auf die proinflammatorischen Mediatoren im Stoffwechsel und hat wahrscheinlich auch antioxidative Wirkungen [3]. In der Volksmedizin wird die Teufelskralle seit langem verwendet. Die Indikationen sind breit gefächert. Sie reichen von der Behandlung von Magenbeschwerden bis hin zur Therapie von Allergien. Die Pflanze regt die Produktion von Magenund Gallensaft an. Die wirksamsten Bestandteile sind die Iridoidglykoside, wie Harpagosid, Harpagid oder Procumbid. Verschiedene Flavonoide, Phenylethanoidglykoside und Triterpene finden sich ebenfalls in der Pflanze. Sie wirken entzündungshemmend und sind schwach schmerzstillend. Sie werden bei Arthrose und anderen rheumatischen Beschwerden eingesetzt. Die Harpagoside sind die

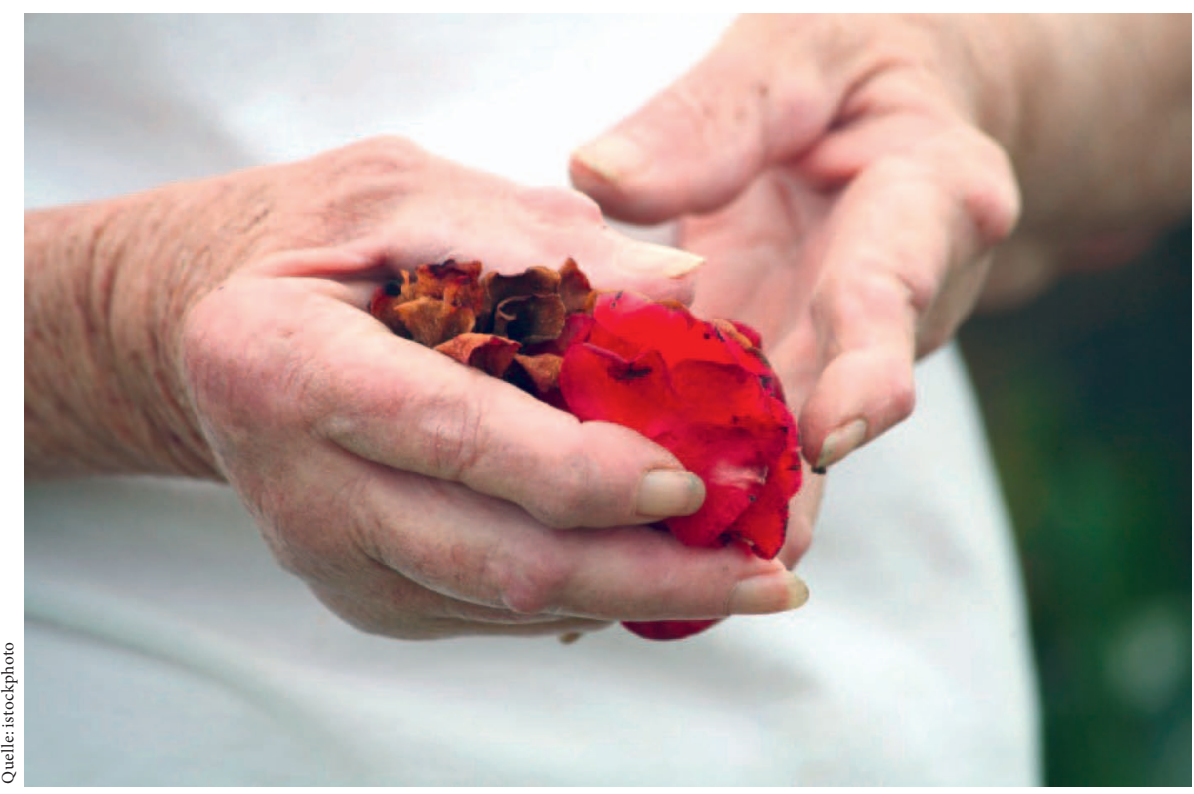

wahrscheinlich entscheidenden Inhaltsstoffe. Es sind monoterpene Glucoside. Sie hemmen die von Liposacchariden induzierte Cox-2-Expression, indem sie Kappa B unterdrücken, das für den Ablauf der Synthese benötigt wird [1]. Die Wirksamkeit der Therapie mit der Teufelskralle ist in verschiedenen Studien untersucht worden. Es zeigt sich, dass der Effekt von der Dosis abhängig ist und $\mathrm{ab}$ einer Dosierung von $\geq 60 \mathrm{mg}$ pro Tag auftritt [4].

Sehr wirksam sind die Inhaltsstoffe von Tripterygium [5]. In einer Cochrane-Studie wurden 3 Studien analysiert, bei denen Tripterygium wilfordii mit Placebo bzw. einer Kontrollgruppe, die Sulfasalazin bekommen hatte, verglichen wurde. Dabei konnte gezeigt werden, dass die Symptome von rheumatischer Arthritis bei einer Tripterygium-Behandlung tatsächlich zurückgingen. Allerdings zeigten sich gravierende Nebenwirkungen, sodass die Cochrane-Autoren den Einsatz von Wirkstoffen aus dieser Pflanze nicht empfehlen [6]. Der Extrakt aus Tripterygium hemmt die Synthese von Zytokinen und anderen Mediatoren, die von Phagozyten ausgeschüttet werden. Auf diese Art und Weise blockieren die Substanzen der Pflanze eine ganze Reihe von Genen, die bei der Entstehung von Entzündungen eine Rolle spielen. Dazu gehören neben Cox-2 auch TNFAlpha, Interferon-Gamma, Interleukin-2, verschiedene Prostaglandine oder iNOS (induzierbare Stickstoffmonoxid-Synthase) [1].

Phytodolor ${ }^{\circledR}$ ist ein phytotherapeutisches Arzneimittel, das ein Wirkstoffgemisch enthält, das aus Blättern der Espe, der Rinde von Pappel und Esche sowie aus der Goldrute (Solidago virgaurea) gewonnen wird. Es hat eine entzündungshemmende und antiödematöse Wirkung und besitzt Eigenschaften als Analgetikum. Es wird bei akuten und subakuten rheumatischen Beschwerden eingesetzt. Das Wirkungsspektrum soll grösser als das der herkömmlichen Antirheumatika sein. Phytodolor wird von einigen Autoren als Alternative zu nichtsteroidalen Antirheumatika (NSAR) und zu den Cox2-Inhibitoren, wie etwa Rofecoxib, betrachtet [7].

Es gibt etwa 30 klinische Studien mit über 1150 Patienten, an denen die Wirkung von Phytodolor untersucht wurde. In einer Studie wurde Phytodolor doppelblind gegen Placebo und offen gegen Indometacin getestet. Es wirkte besser als Placebo und war vergleichbar mit Indometacin. In der 
Verträglichkeit schnitt es allerdings besser als Indometacin ab.

Phytodolor greift durch die Vielzahl der Wirkstoffe, die in dem Arzneimittel vorhanden sind, in den gleichen Mechanismus der Entstehung einer Entzündung ein, wie es auch der phytotherapeutische Wirkstoff von $H$. procumbens tut. Inhaltsstoffe der Espe sind Phenylglykoside wie Salicin oder Salicortin und Flavonoide. Aus der Esche kommen Cumarinderivate hinzu, wie z.B. Esculetin. Aus der Goldrute stammen neben Flavonoiden und der Salicylsäure auch Triterpensaponine wie etwa die Oleanolsäure.

Tee aus Weidenrinde soll schmerzlindernd bei rheumatischen Erkrankungen wirken. Drei- oder viermal täglich eine Tasse aus 2-3 Gramm geschnittener oder pulverisierter Rinde wird empfohlen. Der Gerbstoffgehalt kann vermindert werden, wenn man den Tee kalt ansetzt und dann zum Kochen bringt. Im Handel ist eine Reihe von Weidentee-Mischungen der verschiedenen Anbieter verfügbar, die viele Patienten auch zur Selbstmedikation einsetzen.

Obwohl die Wirkung der Boswelliasäuren, einer Gruppe pentazyklischer Terpene, nicht endgültig gesichert ist, zählen auch die Wirkstoffe des Weihrauchs zum therapeutischen Repertoire, das bei rheumatischen Erkrankungen eingesetzt wird. In der indischen Medizin wird Weihrauch zur Behandlung chronisch rheumatischer Entzündungen verwendet. Als Handelspräparat sind Kapseln für die Verwendung in der ayurvedischen Medizin erhältlich. Die Wirkstoffe des Weihrauchs hemmen die Bildung von Leukotrienen durch nichtkompetitive, reversible Hemmung der 5-Lipoxigenase [5].
Präparate aus Cayennepfeffer enthalten eine Reihe von Wirkstoffen, wie etwa Carotenoide, Vitamin C, Flavonoide oder Steroidsaponine. Wenn sie auf die Haut aufgetragen werden, erregen sie die nozizeptiven Neuronen, die für die Freisetzung der Substanz P, von CGRP (Calcitonin Gene-Related Peptide), Somatostatin und verschiedenen vasoaktiven Polypeptiden verantwortlich sind. In der Folge kommt es zu einer Schmerzunempfindlichkeit, die durch einen Mangel an Substanz P hervorgerufen wird. Entsprechend wirken die Cayennepfeffer-Präparate analgetisch und werden bei rheumatoiden $\mathrm{Be}$ schwerden eingesetzt. Sie werden äusserlich als Pflaster oder als Auflagen auf die schmerzenden Gelenke appliziert. Sie sollten allerdings am gleichen Ort nur 2 Tage lang und erst nach einer Pause von 14 Tagen wieder eingesetzt werden. Die «scharfen» Inhaltsstoffe des Cayennepfeffers führen zu einer starken Reizung der Schleimhäute und sollten auch bei verletzter Haut nicht aufgetragen werden. Im Handel sind einige Präparate erhältlich, wie etwa Pflaster oder auch Kneipp-Bäder mit Cayennepfeffer als Inhaltsstoff.

Die Brennnessel (Urtica) ist eine alte Heilpflanze, die zur Behandlung rheumatischer Beschwerden verwendet wird. Sie enthält Flavonoide, Phytosterole, Derivate der Hydroxy-Zimtsäure und Silikate. Sie wird äusserlich als Tinktur und innerlich als Tee verwendet. Auf dem Markt sind verschiedene phytotherapeutische Präparate, die die Therapie von rheumatischen Beschwerden unterstützen. Sie werden von den meisten Patienten als Ergänzung zur Therapie genommen, die vom Arzt verordnet wird.
Zu Präparaten, die aus den Inhaltsstoffen von Hagebutten der Hundsrose (Rosa canina) gewonnen werden, wird bei der Osteoarthritis gegriffen. Es gibt leichte Hinweise, dass die Wirkstoffe einen therapeutischen Effekt haben [8].

Als wirksam gelten auch die Inhaltsstoffe von Uncaria tomentosa und Uncaria guianensis, die in der Volksmedizin für eine Reihe von Indikationen eingesetzt werden, die mit Entzündungen im Zusammenhang stehen. Die Wirkung geschieht wahrscheinlich über eine Hemmung von TNF-Alpha. Den Wirkstoffen wird zudem eine antioxidative Wirkung zugesprochen [9]. (rfi)

\section{Literatur}

1 Setty AR, Sigal LH: Herbal medications commonly used in the parctice of rheumatology medicine. Semin Arthritis Rheum 2005;34:773784.

2 Cameron M, Gagnier JJ, Chrubasik S: Herbal therapy for treating rheumatoid arthritis. Cochrane Database Syst Rev 2011;2:CD002948.

3 Denner SS: A review of the efficacy and safety of devil's claw for pain associated with degenerative musculoskeletal diseases, rheumatoid, and osteoarthritis. Hollist Nurs Pract 2007;21:203207.

4 Gagnier JJ, Chrubasik S, Manheimer E: Harpagophytum procumbens for osteoarthritis and low back pain: a systematic review. BMC Complement Altern Med 2004;4:13.

5 Goldbach-Mansky R, Wilson M, Fleischmann R, Olsen N, Silverfield J, Kempf P, Kivitz A, Sherrer Y, Pucino F, Csako G, Costello R, Pham TH, Snyder C, van der Heijde D, Tao $\mathrm{X}$, Wesley R, Lipsky PE: Comparison of Tripterygium wilfordii Hook $\mathrm{F}$ versus sulfasalazine in the treatment of rheumatoid arthritis: a randomized trial. Ann Int Med 2009;18: 228-240.

6 Tao X et al: Herbs at a glance: Thunder God Vine. Phytomedicine 2006;13:371-377.

7 Gundermann KJ, Müller J: Phytodolor - effects and efficacy of a herbal medicine. Wien Med Wochenschr 2007;157:343-347.

$>8$ Chrubasik C, Duke RK, Chrubasik S: The evidence for clinical efficacy of rose hip and seed: a systematic review. Phytother Research 2006;20:1-3.

9 Hardin SR: Cat's claw: an Amazonian vine decreases inflammation in osteoarthritis. Compl Ther Clin Pract 2007;13:25-28. 